Pacific Journal of Mathematics

ON THE CHARACTERIZATION OF AN INTERESTING 


\title{
ON THE CHARACTERIZATION OF AN INTERESTING PROPERTY OF THE ARCSIN DISTRIBUTION
}

\author{
J. H. B. KeMPERMAN AND M. SKIBINSKY
}

The property that product and sum of independent identically distributed random variables are equidistributed is characterized when the original distribution has all moments. This equidistribution property is shown to relate in a natural way to the uniform distribution on a compact group. The result proves a conjecture due to Norton.

Introduction. By the notation $U \sim V$ we will indicate that the random variables $U$ and $V$ are identically distributed. We are concerned with the property

$$
Z_{1} Z_{2} \sim Z_{1}+Z_{2}
$$

where $Z, Z_{1}, Z_{2}$ are independent and identically distributed. All our comments and results assume that all the moments

$$
d_{i}=E Z^{i}, \quad(i=0,1,2, \cdots)
$$

exist. We do not know if any distribution (or random variable $Z$ ) has property $(*)$ but does not possess all moments.

Clearly, (*) implies that

$$
d_{n}^{2}=\sum_{k=0}^{n}\left(\begin{array}{l}
n \\
k
\end{array}\right) d_{k} d_{n-k}, \quad(n=0,1,2, \cdots) .
$$

Equivalently,

$$
\left(d_{n}-1\right)^{2}=1+\sum_{k=1}^{n-1}\left(\begin{array}{l}
n \\
k
\end{array}\right) d_{k} d_{n-k}, \quad(n=1,2, \cdots) .
$$

As was observed by Norton (1978), (2) implies that $-2 \leqq Z \leqq 2$. After all, since $\left|d_{k}\right|^{1 / k} \leqq\left(d_{2 n}\right)^{1 / 2 n},(2)$ implies $d_{2 n}^{2} \leqq 2^{2 n} d_{2 n}$, thus, $E|Z|^{2 n} \leqq$ $2^{2 n}$ for all $n$. Since the distribution of a bounded random variable is completely determined by its moment sequence, condition (3) is both necessary and sufficient for $(*)$.

It was shown by Norton (1975) that (*) holds for the Arcsin distribution on $[-2,+2]$, that is, when $Z$ is distributed according to the probability density

$$
f(z)=\pi^{-1}\left(4-z^{2}\right)^{-1 / 2} \text { for } \quad-2 \leqq z \leqq+2,
$$

$(f(z)=0$, otherwise). The moments of this distribution are 


$$
d_{2 m}^{*}=\left(\begin{array}{c}
2 m \\
m
\end{array}\right) ; \quad d_{2 m+1}^{*}=0, \quad(m=0,1,2, \cdots) .
$$

Norton verified $(*)$ by showing that (2) holds when $d_{i}=d_{i}^{*}$.

Relation (3) may be used to recursively calculate the moments $d_{n}$. For $n=1$, one has $\left(d_{1}-1\right)^{2}=1$ so that $d_{1}=0$ or $d_{1}=2$. Since $Z \leqq 2$, the latter leads to a degenerate solution of (*), namely,

$$
Z=2 \text {. }
$$

If $d_{1}=0$ then (3) yields $\left(d_{2}-1\right)^{2}=1$ so that $d_{2}=0$ or $d_{2}=2$. The case $d_{2}=E Z^{2}=0$ leads to the degenerate solution

$$
Z=0
$$

of $(*)$. If $d_{1}=0, d_{2}=2$ then $(3)$ yields $\left(d_{3}-1\right)^{2}=1$ so that $d_{3}=0$ or $d_{3}=2$. Merely assuming that $d_{1}=0, d_{2}=2$, it follows from (3) that $\left(d_{4}-1\right)^{2}=1+8 d_{1} d_{3}+6 d_{2}^{2}=25$, hence, $d_{4}=6$. If $d_{1}=d_{3}=0$ then $\left(d_{5}-1\right)^{2}=1+10 d_{1} d_{4}+20 d_{2} d_{3}=1$ so that $d_{5}=0$ or $d_{5}=2$. And so on.

If one insists upon a distribution which is symmetric about zero, then of necessity $d_{2 k+1}=0$ for all $k$ and the above calculations yield that $d_{k}=d_{k}^{*}$ for all $k$. This shows that the Arcsin law is the only symmetric distribution which satisfies (*).

Setting aside the Arcsin distribution and the degenerate solutions (6) and (7), it follows from the above recursive scheme that to any other solution $\left\{d_{k}\right\}$ of (3) there corresponds a unique positive integer $m$ such that

$$
d_{k}=d_{k}^{*} \quad \text { for } k=0,1, \cdots, 2 m, 2 m+2 ; \quad d_{2 m+1}=2 ;
$$

(in particular, $d_{1}=d_{3}=\cdots=d_{2 m-1}=0$ ). With the $d_{j}$ as in (8), Norton (1978) showed that the Hankel determinant

$$
\Delta_{m+1}=\operatorname{det}\left(d_{i+j} ; i, j=0,1, \cdots, m+1\right)
$$

is equal to zero for $m \leqq 14$. Afterwards, Shantaram (1978) established the same for all $m \geqq 1$.

Let $Z$ be any random variable whose moments satisfy (8). It follows from $\Delta_{m+1}=0$ that there exist numbers $a_{0}, a_{1}, \cdots, a_{m}$ not all zero such that $E\left(\sum_{j=0}^{m+1} a_{j} z^{j}\right)^{2}=0$. Equivalently, the support of $Z$ is a finite set consisting of at most $m+1$ points. It follows from well-known results (see Karlin and Studden (1966), page 42) that the distribution of $Z$ on hand (if it exists) is completely determined by the moments (8).

Note that the Arcsin law is the only nondiscrete solution of (*). As to the discrete solutions, Norton (1978) conjectured the following. 
Conjecture. Let $m$ be any positive integer. Then there does exist a random variable $Z(m)$ whose moments satisfy (8). Moreover, the support of $Z(m)$ consists of precisely $m+1$ points. Finally, this random variable $Z(m)$ has property $(*)$.

The conjecture turns out to be correct. More precisely, we shall prove the following.

THEOREM 1. Let $Z$ be a random variable having all moments. Then in order that $Z$ satisfies (*), it is necessary and sufficient that one of the following is true.

(i) $Z$ has the Arcsin distribution (4);

(ii) $Z=0$;

(iii) there exists a nonnegative integer $m$ such that $Z$ is distributed as $Z(m)$, where

$$
\operatorname{Pr}(Z(m)=2) \quad=1 /(2 m+1) ;
$$

$$
\operatorname{Pr}\left(Z(m)=2 \cos \frac{2 j \pi}{2 m+1}\right)=2 /(2 m+1) \quad \text { for } \quad j=1,2, \cdots, m \text {. }
$$

The result due to Shantaram that $\Delta_{m+1}=0$ is not used in the proof. As will be seen, the solutions (4) and (9) of (*) arise in a very natural way from the properties of a uniformly distributed random variable $U$ taking values in a compact group.

One may ask how far the above considerations carry over to a relation of the form

$$
Z_{1} Z_{2} \cdots Z_{r} \sim Z_{1}+Z_{2}+\cdots+Z_{s},
$$

where the $Z_{j}$ are independent, each distributed as $Z=Z_{1}$. Here, $r \geqq 2$ and $s \geqq 2$ are fixed integers. Provided all moments $d_{n}=E Z^{n}$ exist, one easily shows that necessarily $-C \leqq Z \leqq C$, where $C=s^{1 /(r-1)}$. There are always the trivial solutions $Z=0$ and $Z=C$, and further $Z=-C$ in the case $r$ is odd. We conjecture that no other solutions exist, unless $r=s=2$.

REMARK. After the present paper was completed, Professor Govind Mudholkar kindly showed us a copy of the paper "On the stochastic equation $X+Y=X Y$ ", by R. Shantaram (presented at the International Summer School on Statistical Distributions in Scientific Work, Trieste, Summer 1980). Shantaram's paper is devoted to a proof of exactly the above Theorem 1 , but by a very different reasoning.

2. Random variables taking values in a compact group. Let $G$ be a compact abelian group, taking addition as the group operation. By a character $g$ of $G$ we mean a complex-valued function on 
$G$ which defines a continuous homomorphism of $G$ into the circle group $\{z:|z|=1\}$. In particular,

$$
g\left(x+x^{\prime}\right)=g(x) g\left(x^{\prime}\right) ; \quad|g(x)|=1 ; \quad g(0)=1 .
$$

Each character $g$ of $G$ will be labeled by an element $y$ of an index set $\hat{G}$ and written as $g(x)=(x, y)$, The set $\hat{G}$ is made into a (discrete) additive abelian group by defining $\left(x, y+y^{\prime}\right)=(x, y)\left(x, y^{\prime}\right)$. One has $(x, 0)=1$ and $(-x, y)=(x,-y)$.

The Haar measure on $G$ is denoted by $m(\cdot) ; m(G)=1$. Let $U$ be a random variable taking values in $G$. It is said to be uniformly distributed on $G$ when $\operatorname{Pr}(U \in B)=m(B)$ for each Borel subset $B$ of $G$. This happens if and only if

$$
E(U, y)=0 \text { for each } y \in \hat{G} \text { with } y \neq 0 .
$$

Next, let $U_{1}, U_{2}$ be random variables taking values in $G$. They are independent, each uniformly distributed on $G$, if and only if

$$
E\left[\left(U_{1}, y_{1}\right)\left(U_{2}, y_{2}\right)\right]=0 \text { whenever }\left(y_{1}, y_{2}\right) \neq(0,0) \text {. }
$$

Suppose this is true and consider

$$
V_{1}=U_{1}+U_{2} ; \quad V_{2}=U_{1}-U_{2} .
$$

Then

$$
\left(V_{1}, y_{1}\right)\left(V_{2}, y_{2}\right)=\left(U_{1}, y_{1}\right)\left(U_{2}, y_{1}\right)\left(U_{1}, y_{2}\right)\left(U_{2},-y_{2}\right)=\left(U_{1}, y_{1}+y_{2}\right)\left(U_{2}, y_{1}-y_{2}\right) \text {. }
$$

Using the criterion (12) applied to the pair $V_{1}, V_{2}$, it follows that $V_{1}, V_{2}$ are independent, each uniformly distributed, if and only if $\left(y_{1}, y_{2}\right) \neq(0,0)$ implies that $\left(y_{1}+y_{2}, y_{1}-y_{2}\right) \neq(0,0)$. Equivalently, the character group $\hat{G}$ contains no elements $y$ of order 2, (that is, $y \neq 0$ and $2 y=0$ ). Equivalently, $G$ does not have any closed subgroup $H$ with $m(H)=1 / 2$, (that is, $H$ is of index 2); (if such a subgroup $H$ does exist then $V_{1}-V_{2} \in H$ showing that $V_{1}, V_{2}$ would be dependent). The above remarks yield the following result.

LemMa 1. Let $G$ be a compact abelian group and let $U_{1}, U_{2}$ be independent copies of a random variable $U$ taking values in $G$. Further consider the random variables

$$
S_{1}=h\left(U_{1}, U_{2}\right) ; \quad S_{2}=h\left(U_{1}+U_{2}, U_{1}-U_{2}\right),
$$

where $h$ is any measurable function on $G \times G$ taking values in some measurable space.

We assert that $S_{1}$ and $S_{2}$ are identically distributed as soon as $U$ is uniformly distributed on some closed subgroup $G_{1}$ of $G$ with the property that $G_{1}$ possesses no closed subgroup $H_{1}$ of index 2. 
In applying Lemma 1 , we shall take $G$ as the additive group of real numbers modulo $2 \pi$. It will be convenient to regard $G$ as the additive group of real numbers, identifying numbers $x^{\prime}$ and $x^{\prime \prime}$ such that $x^{\prime} \equiv x^{\prime \prime}(\bmod 2 \pi)$. A function on $G$ can be represented by a function on $R$ which is periodic with period $2 \pi$. Similarly, for a function $h\left(x_{1}, x_{2}\right)$ on $G \times G$.

THEOREM 2. Let $U$ be a real random variable, $0 \leqq U \leqq 2 \pi$, and put

$$
Z=2 \cos U \text {. }
$$

We assert that $Z$ has property (*) in each of the following cases.

(i) $U$ is uniformly distributed on $[0,2 \pi]$. In this case, $Z$ defined by (15) has the Arcsin distribution (4).

(ii) For some nonnegative integer $m$, the random variable $U$ is uniformly distributed on the finite set $\{2 \pi j /(2 m+1), j=0,1, \cdots, 2 m\}$. In this case,

$$
\begin{array}{ll}
\operatorname{Pr}(Z=2) & =1 /(2 m+1) ; \\
\operatorname{Pr}\left(Z=2 \cos \frac{2 \pi j}{2 m+1}\right)=2 /(2 m+1) \quad \text { for } \quad j=1, \cdots, m .
\end{array}
$$

Proof. The only proper closed subgroups of the additive group $G$ of real numbers modulo $2 \pi$ are the cyclic subgroups

$$
C_{n}=\{2 \pi j / n, j=0,1, \cdots, n-1\},
$$

$(n=1,2, \cdots)$. Hence, the last condition of Lemma 1 is satisfied if and only if either $G_{1}=G$ or $G_{1}=C_{2 m+1}(m=0,1, \cdots)$.

Let $G_{1}$ be one of the latter groups and suppose $U$ is uniformly distributed on $G_{1}$. Let $U_{1}, U_{2}$ be independent copies of $U$. It follows from Lemma 1 that the random variables $S_{1}, S_{2}$ defined by (14) are always identically distributed. Choosing $h\left(x_{1}, x_{2}\right)=2 \cos x_{1}+$ $2 \cos x_{2}$, we find that

$$
S_{1}=h\left(U_{1}, U_{2}\right)=2 \cos U_{1}+2 \cos U_{2}=Z_{1}+Z_{2}
$$

has the same distribution as

$$
S_{2}=2 \cos \left(U_{1}+U_{2}\right)+2 \cos \left(U_{1}-U_{2}\right)=4 \cos U_{1} \cos U_{2}=Z_{1} Z_{2} .
$$

In other words, $Z=2 \cos U$ has property (*). Calculating the distribution of $Z$ in each case, one arrives at Theorem 2.

REMARK. Note that the sufficiency part of Theorem 1 is hereby proved. It is easy to verify directly that (16) defines a random variable that has property $(*)$. Put 


$$
z_{j}=2 \cos \left(\frac{2 \pi j}{2 m+1}\right), \quad\left(z_{-j}=z_{j}\right)
$$

It suffices to verify that, for any function $g$,

$$
E g\left(Z_{1}+Z_{2}\right)=(2 m+1)^{-2} \sum_{j=-m}^{m} \sum_{k=-m}^{m} g\left(z_{j}+z_{k}\right)
$$

is equal to

$$
E g\left(Z_{1} Z_{2}\right)=(2 m+1)^{-2} \sum_{j=-m}^{m} \sum_{k=-m}^{m} g\left(z_{j} z_{k}\right) .
$$

From $(17), z_{j}+z_{k}=z_{r} z_{s}$ when both $j \equiv r+s(\bmod 2 m+1)$ and $k \equiv$ $r-s(\bmod 2 m+1)$. Equivalently, when $2 r \equiv j+k(\bmod 2 m+1)$ and $2 s \equiv j-k(\bmod 2 m+1)$. This one-one correspondence between pairs $(j, k)$ and $(r, s)$ yields the desired result.

3. Proof of necessity in Theorem 1. We are concerned with random variables $Z$ which have property $(*)$ and further possess all moments $d_{k}=E Z^{k}$. We may assume that $\operatorname{Pr}(Z \neq 0)>0$ and that $Z$ does not have the Arcsin distribution. As we have seen, this implies that $-2 \leqq Z \leqq+2$ and further that $\left\{d_{k}\right\}$ satisfies (8) for some unique nonnegative integer $m$.

For each integer $m \geqq 0$, let $K_{m}$ denote the class of all distributions (probability measures) on $[-2,+2]$ such that the corresponding moment sequence $\left\{d_{k}\right\}$ satisfies (8). Clearly, $K_{0}$ contains only the degenerate distribution at $z=2$. It remains to prove that the only distributions in $\bigcup_{m=0}^{\infty} K_{m}$ are the distributions of the random variables $Z(0), Z(1), \cdots$ defined by (9).

Let $\mu_{m}$ denote the distribution of $Z(m)$. Its support is of the form

$$
A(m)=\left\{z_{m, j}=2 \cos (2 \pi j /(2 m+1)) ; j=0,1, \cdots, m\right\} .
$$

We know by Theorem 2 that $Z(m)$ has property (*), hence, $\mu_{m}$ belongs to some unique class $K_{M}$. We claim that $M \leqq m$.

For, otherwise, from (8) with $m$ replaced by $M$, the moments $d_{k}$ of $Z(m)$ satisfy $d_{1}=d_{3}=\cdots=d_{2 m-1}=d_{2 m+1}=0$. Equivalently,

$$
\sum_{j=0}^{m} p_{j}\left(z_{m, j}\right)^{2 k-1}=0 \quad \text { for } \quad k=1,2, \cdots, m+1,
$$

where $p_{j}=\operatorname{Pr}\left(Z(m)=z_{m, j}\right)$. Note that $z_{m, h} \neq-z_{m, j}$, in fact, the $z_{m, j}^{2}(j=0,1, \cdots, m)$ are distinct and nonzero. But then the above system would imply that $p_{j}=0$ for all $j$.

We conclude that

$$
\left\{\mu_{0}, \mu_{1}, \cdots, \mu_{m}\right\} \subset \bigcup_{M=0}^{m} K_{M}, \quad(m=0,1,2, \cdots) .
$$


This shows that it suffices to prove that each class $K_{M}$ contains at most one distribution. Since all the $\mu_{m}$ are different, this would imply that $K_{m}=\left\{\mu_{m}\right\}$ for all $m$.

There are many ways of showing that $K_{m}$ consists of at most a single distribution. As observed in the paragraph following (8), this follows for instance from Shantaram's (1978) result that $\Delta_{m+1}=0$.

As an independent proof, we even claim that there can be at most one distribution $\mu$ on $[-2,+2]$ which satisfies

$$
d_{2 k-1}=0 \text { for } k=1, \cdots, m ; d_{2 m+1}=2 .
$$

Here, $m$ is a fixed nonnegative integer (and nothing is assumed about the moments $d_{2 k}$ ). Namely, consider the polynomial

$$
f(z)=1+T_{2 m+1}(-z / 2)=1+a_{0} z+a_{1} z^{3}+\cdots+a_{m} z^{2 m+1} .
$$

Here, $T_{n}(x)$ denotes the $n$th Tchebycheff polynomial. For $x \in$ $[-1,+1]$, it is defined by $T_{n}(x)=\cos n \theta$ where $\cos \theta=x$. Writing $\cos n \theta$ in complex form, we see that the leading coefficient in $T_{n}(x)$ equals $2^{n-1}$. Therefore, the leading coefficient $a_{m}$ of $f(z)$ equals

$$
a_{m}=2^{2 m}(-1 / 2)^{2 m+1}=-1 / 2 .
$$

Integrating $f(z)$ relative to a measure $\mu$ satisfying (19), it follows that

$$
\int f(z) \mu(d z)=1+a_{m} d_{2 m+1}=0 .
$$

Since obviously $f(z) \geqq 0$ on $[-2,+2]$, we conclude that $\mu$ must be supported by $Z(f)=\{z: f(z)=0\}$. It is easily seen that $Z(f)$ is precisely the set $A(m)$ defined by (18). Condition (19) then implies that $q_{j}=\mu\left(\left\{z_{m, j}\right\}\right)$ satisfies the system of $m+1$ equations

$$
\sum_{j=0}^{m} q_{j}=1 ; \quad \sum_{j=0}^{m} q_{j}\left(z_{m, j}\right)^{2 k-1}=0 \quad \text { for } \quad k=1, \cdots, m .
$$

Since the corresponding matrix has rank $m+1$, there can be at most one solution.

4. Related problems. The referee kindly pointed to the related paper by Arnold and Groeneveld (1980). It contains the following results.

(A) If the random variable $X$ is symmetric (about 0 ) then

$$
X^{2} \sim(1+X) / 2
$$

if and only if $X$ has the Aresin distribution on $[-1,+1]$. The corresponding density is 


$$
f(x)=\pi^{-1}\left(1-x^{2}\right)^{-1 / 2} \text { for }-1<x<1 .
$$

(B) Suppose $-1 \leqq X \leqq+1$ and further that both $X$ and $X^{2}-1 / 2$ are symmetric. Then

$$
2 X\left(1-X^{2}\right)^{1 / 2} \sim X
$$

if and only if $X$ has the Arcsin distribution on $[-1,+1]$.

(C) Let $X, X_{1}, X_{2}$ be i.i.d. and suppose that both $X$ and $X^{2}-1 / 2$ are symmetric. Then

$$
X_{1}^{2}-X_{2}^{2} \sim X_{1} X_{2}
$$

if and only if $X$ has the Arcsin distribution on $[-1,+1]$.

The proofs are easy. Simple counterexamples show that the above symmetry conditions cannot be omitted.

It would be desirable to characterize the properties (21), (22) and (23) without making any symmetry assumption. Without proof, we mention the following results.

(A1) If $X$ satisfies (21) then necessarily $-1 \leqq X \leqq+1$. Thus, one may write $X=\cos \pi Y$ with $0 \leqq Y \leqq 1$. Property (21) is equivalent to $T Y \sim Y$, where

$$
T y=1-2|y-1 / 2|,
$$

thus, $T$ defines a continuous map of $[0,1]$ into itself.

(A2) Suppose $X$ satisfies (21) and, moreover, has an absolutely continuous distribution (relative to Lebesgue measure). Then $Y$ has the uniform distribution on $[0,1]$, equivalently, $X$ has the Arcsin distribution on $[-1,+1]$.

(A3) We conjecture that (21) admits at least one solution $X$ having a purely singular distribution (with no atoms).

(A4) Suppose the random variable $X$ has a finite support. Then $X$ satisfies (21) if and only if its distribution is a convex linear combination of the uniform distributions on the different (mutually disjoint) finite closed orbits of the continuous transformation $S x=$ $2 x^{2}-1$ of $[-1,+1]$ into itself.

A number $x \in[-1,+1]$ belongs to such an orbit if and only if $x=\cos \pi y$ with $y=h /(2 m+1)$ as a rational number with odd denominator, $(h \in\{0,1, \cdots, 2 m\})$. Besides $y=1$, the excluded rational values $y$ (such as $1 / 2$ and $1 / 6$ ) are precisely those whose expansion $y=\cdot y_{1} y_{2} \cdots$ to the base 2 is not purely periodic.

(A5) Let $m$ be a nonnegative integer and put $d=(2 m+1)^{-1}$. Then $X=\cos \pi(U+j d / 3)$ satisfies $(21)$ as soon as $j \in\{0,1,2\}$ while $U$ has a uniform distribution on $\{h d ; h=0,1, \cdots, 2 m\}$.

(B1) Suppose $X$ satisfies $-1 \leqq X \leqq+1$ and (22). Then $W=$ $1-2 X^{2}$ satisfies 


$$
W^{2}=1-4 X^{2}\left(1-X^{2}\right) \sim 1-X^{2}=(1+W) / 2,
$$

and we are back to Problem A.

(B2) Given $-1 \leqq X \leqq+1$, one may write $X=U \sin \pi Y / 2$ where either $U= \pm 1,0<Y \leqq 1$ or $U=0, Y=0$. Condition (22) says precisely that $U \sin \pi Y / 2 \sim U \sin \pi Y$. Equivalently, $(U, Y) \sim(U, T Y)$ with $T$ as in (24). That is, property (22) holds if and only if each of the measures

$$
\mu_{\varepsilon}(A)=\operatorname{Pr}(U=\varepsilon, Y \in A), \quad(\varepsilon=+1 \text { or }-1)
$$

is invariant under $T$.

(B3) Suppose $X$ satisfies (22) and, moreover, is absolutely continuous. Then each of the measures $\mu_{\varepsilon}$ is a multiple of the uniform distribution on $[0,1]$, in particular, $U$ and $Y$ are independent. The present density of $X$ is of the form

$$
\begin{array}{rlrr}
f(x) & =c_{1}\left(1-x^{2}\right)^{-1 / 2} & \text { for } & 0<x<1 ; \\
& =c_{2}\left(1-x^{2}\right)^{-1 / 2} & \text { for } & -1<x<0,
\end{array}
$$

with the $c_{i}$ as nonnegative constants, $\left(c_{1}+c_{2}=2 / \pi\right)$.

(C) Let $X$ satisfy (23). One easily sees that $E X^{2 k+1}=0 \quad(k=$ $0,1, \cdots)$, in so far these moments exist. Hence, if $X$ is also bounded then it must be symmetric.

Condition (23) is satisfied by $X=c \sin 2 \pi Y$, where $c \geqq 0$ is constant and $Y$ is uniform on a set $\{h /(2 m+1) ; h=0,1, \cdots, 2 m\}$. In the limit $m \rightarrow \infty$, this gives rise to the Arcsin distribution on $[-c,+c]$. We conjecture that $(23)$ has no other solutions.

\section{REFERENCES}

1. B. C. Arnold and R. A. Groeneveld, Some properties of the Arcsin distribution, J. Amer. Statist. Assoc., 75 (1980), 173-175.

2. S. Karlin and W. Studden, Tchebycheff Systems: With Applications in Analysis and Statistics, Interscience, New York, 1966.

3. R. M. Norton, On properties of the Arcsin law, Sankhyā, Series A 37 (1975), 306-308.

4. - Moment properties and the Arcsin law, Sankyā, Series A 40 (1978), 192-198.

5. R. Shantaram, A characterization of the Arcsin law, Sankhyā, Series A 40 (1978), 199-207.

6. - On the stochastic equation $X+Y=X Y$, Manuscript no. 62. International Summer School on Statistical Distributions in Scientific Work, (1980), Trieste.

Received November 11, 1980. This research was supported in part by the National Science Foundation, contract 7703620 .

UNIVERSITY OF ROCHESTER

ROCHESTER, NY 14627

AND

UNIVERSITY OF MASSACHUSETTS

AMHERST, MA 01003 



\title{
PACIFIC JOURNAL OF MATHEMATICS
}

\section{EDITORS}

\author{
DONALD BABBITT (Managing Editor) \\ University of California \\ Los Angeles, CA 90024 \\ Hugo Rossi \\ University of Utah \\ Salt Lake City, UT 84112 \\ C. C. MOORE and ARthur Agus \\ University of California \\ Berkeley, CA 94720
}

J. DUGUNDJI

Department of Mathematics

University of Southern California

Los Angeles, CA 90007

R. FINN and J. MILGRAM

Stanford University

Stanford, CA 94305

\section{ASSOCIATE EDITORS}
R. ARENS
E. F. BeCKENBACH
B. H. NeumanN
F. WoLF
K. YoSHIDA

\section{SUPPORTING INSTITUTIONS}

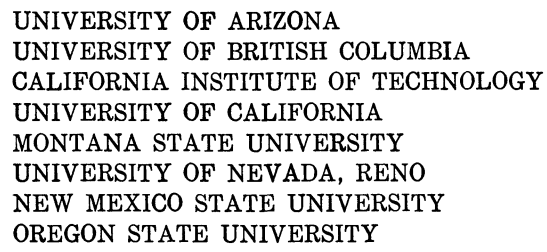

UNIVERSITY OF ARIZONA

UNIVERSITY OF BRITISH COLUMBIA

CALIFORNIA INSTITUTE OF TECHNOLOGY

UNIVERSITY OF CALIFORNIA

MONTANA STATE UNIVERSITY

UNIVERSITY OF NEVADA, RENO

NEW MEXICO STATE UNIVERSITY

OREGON STATE UNIVERSITY

\author{
UNIVERSITY OF OREGON \\ UNIVERSITY OF SOUTHERN CALIFORNIA \\ STANFORD UNIVERSITY \\ UNIVERSITY OF AAWAII \\ UNIVERSITY OF TOKYO \\ UNIVERSITY OF UTAH \\ WASHINGTON STATE UNIVERSITY \\ UNIVERSITY OF WASHINGTON
}

The Supporting Institutions listed above contribute to the cost of publication of this Journal, but they are not owners or publishers and have no responsibility for its content or policies,

Mathematical parers intended for publication in the Pacific Journal of Mathematics should be in typed form or offset-reproduced, (not dittoed), double spaced with large margins. Please do not use built up fractions in the text of the manuscript. However, you may use them in the displayed equations. Underline Greek letters in red, German in green, and script in blue. The first paragraph or two must be capable of being used separately as a synopsis of the entire paper. Please propose a heading for the odd unmbered pages of less than 35 characters. Manuscripts, in triplicate, may be sent to any one of the editors. Please classify according to the scheme of Math. Reviews, Index to Vol. 39. Supply name and address of author to whom proofs should be sent. All other communications should be addressed to the managing editor, or Elaine Barth, University of California, Los Angeles, California, 90024.

50 reprints to each author are provided free for each article, only if page charges have been substantially paid. Additional copies may be obtained at cost in multiples of 50 .

The Pacific Journal of Mathematics is issued monthly as of January 1966, Regular subscription rate: $\$ 114.00$ a year (6 Vol., 12 issues). Special rate: $\$ 57.00$ a year to individual members of supporting institution.

Subscriptions, orders for numbers issued in the last three calendar years, and changes of address shoud be sent to Pacific Journal of Mathematics, P.O. Box 969, Carmel Valley, CA 93924, U.S.A. Old back numbers obtainable from Kraus Periodicals Co., Route 100, Millwood, NY 10546.

PUBLISHED BY PACIFIC JOURNAL OF MATHEMATICS, A NON-PROFIT CORPORATION

Printed at Kokusai Bunken Insatsusha (International Academic Printing Co., Ltd.). 8-8, 3-chome, Takadanobaba, Shinjuku-ku, Tokyo 160, Japan. 


\section{Pacific Journal of Mathematics}

\section{Vol. 103, No. $2 \quad$ April, 1982}

Alberto Alesina and Leonede De Michele, A dichotomy for a class of positive

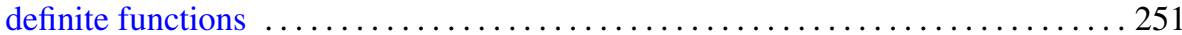

Kahtan Alzubaidy, Rank 2 -groups, $p>3$, and Chern classes . . . . . . . . . . 259

James Arney and Edward A. Bender, Random mappings with constraints on

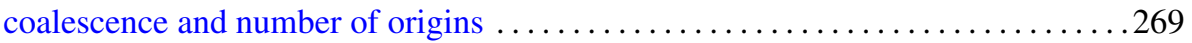

Bruce C. Berndt, An arithmetic Poisson formula . . . . . . . . . . . . . . . 295

Julius Rubin Blum and J. I. Reich, Pointwise ergodic theorems in 1.c.a. groups . . . 301

Jonathan Borwein, A note on $\varepsilon$-subgradients and maximal monotonicity . . . . . . . 307

Andrew Michael Brunner, Edward James Mayland, Jr. and Jonathan Simon,

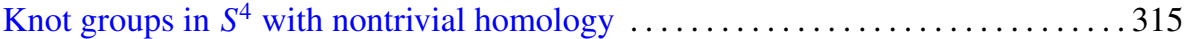

Luis A. Caffarelli, Avner Friedman and Alessandro Torelli, The two-obstacle

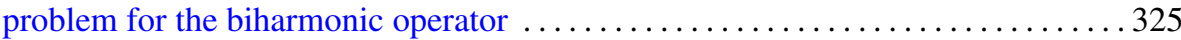

Aleksander Całka, On local isometries of finitely compact metric spaces . . . . . . 337

William S. Cohn, Carleson measures for functions orthogonal to invariant

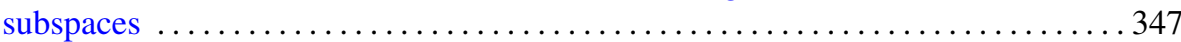

Roger Fenn and Denis Karmen Sjerve, Duality and cohomology for one-relator

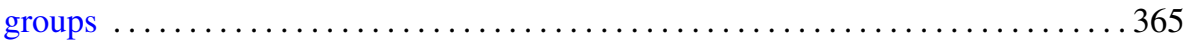

Gen Hua Shi, On the least number of fixed points for infinite complexes . . . . . . . 377

George Golightly, Shadow and inverse-shadow inner products for a class of linear

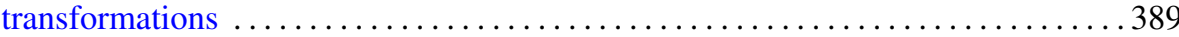

Joachim Georg Hartung, An extension of Sion's minimax theorem with an

application to a method for constrained games $\ldots \ldots \ldots \ldots \ldots \ldots \ldots \ldots \ldots \ldots . \ldots 4$

Vikram Jha and Michael Joseph Kallaher, On the Lorimer-Rahilly and

Johnson-Walker translation planes

Kenneth Richard Johnson, Unitary analogs of generalized Ramanujan sums .

Peter Dexter Johnson, Jr. and R. N. Mohapatra, Best possible results in a class of inequalities

Dieter Jungnickel and Sharad S. Sane, On extensions of nets

Johan Henricus Bernardus Kemperman and Morris Skibinsky, On the

characterization of an interesting property of the arcsin distribution ...

Karl Andrew Kosler, On hereditary rings and Noetherian $V$-rings

William A. Lampe, Congruence lattices of algebras of fixed similarity type. II . . . . 475

M. N. Mishra, N. N. Nayak and Swadeenananda Pattanayak, Strong result for real zeros of random polynomials

Sidney Allen Morris and Peter Robert Nickolas, Locally invariant topologies on free groups

Richard Cole Penney, A Fourier transform theorem on nilmanifolds and nil-theta functions

Andrei Shkalikov, Estimates of meromorphic functions and summability theorems

László Székelyhidi, Note on exponential polynomials

William Thomas Watkins, Homeomorphic classification of certain inverse limit spaces with open bonding maps $\ldots \ldots \ldots \ldots \ldots \ldots \ldots \ldots$

David G. Wright, Countable decompositions of $E^{n}$

Takayuki Kawada, Correction to: "Sample functions of Pólya processes" .

Z. A. Chanturia, Errata: "On the absolute convergence of Fourier series of the 